\title{
Efeitos da fisioterapia respiratória em bebês de risco sob cuidados especiais
}

\author{
Effects of respiratory physiotherapy on babies of risk under special care
}

Efectos de la fisioterapia respiratoria en bebés de riesgo bajo cuidados especiales

\author{
Rayane Priscila Batista DOS SANTOS \\ Adriano LOURENÇO² \\ Luana Fonsêca DOS SANTOS ${ }^{1}$ \\ Ana Isabele Andrade NEVES ${ }^{2}$ \\ Camille Pessoa DE ALENCAR ${ }^{1}$ \\ Yago Tavares PINHEIRO'
}

\author{
${ }^{I}$ Departamento de Fisioterapia. Universidade Federal da Paraíba-UFPB, 58051-900, João Pessoa-PB, Brasil \\ ${ }^{2}$ Faculdade de Ciências da Saúde do Trairí, Programa de Pós-Graduação em Ciências da Reabilitação, Universidade Federal do Rio Grande do Norte-UFRN, \\ 59200-000, Santa Cruz-RN, Brasil
}

\section{Resumo}

Introdução: O recém-nascido $(\mathrm{RN})$ é classificado como prematuro quando apresenta idade gestacional inferior a 37 semanas e peso de nascimento igual ou abaixo de $2.550 \mathrm{~g}$. Devido à imaturidade do sistema respiratório, o neonato está sujeito a apresentar diversas complicações, dentre elas, as respiratórias, o que ocasiona o seu prolongamento na unidade de terapia intensiva neonatal (UTIN). A fisioterapia respiratória é de grande importância no tratamento e recuperação do RN através da aplicação de técnicas de higiene brônquica (HB). O estudo teve como objetivo investigar os efeitos da fisioterapia respiratória no recém-nascido prematuro publicados na literatura científica. Materiais e Métodos: Trata-se de um a revisão integrativa realizada nas bases de dados Biblioteca Virtual em Saúde, LILACS, Medline, SciELO, SCOPUS e ISI Web of Knowledge, incluindo artigos publicados no período de 2007 a 2015. Oito artigos foram incluídos nesta revisão. Resultados e Discussão: A atuação da fisioterapia respiratória foi analisada mediante os efeitos da aplicação das técnicas de HB mais utilizadas no recém-nascido pré-termo (RNPT), podendo destacar a tapotagem, vibrocompressão, drenagem postural e aspiração. Foram realizadas comparações para comprovar a eficácia e os possíveis efeitos colaterais que pudessem alterar o funcionamento da mecânica respiratória do RN. Os estudos mostraram a efetividade da fisioterapia respiratória e os efeitos das manobras na condição respiratória do neonato de risco. Conclusão: A fisioterapia tem um papel importante no cuidado ao recém-nascido pré-termo, mas necessita de mais estudos que comprovem sua eficácia e sua importância na melhora da condição de vida do neonato.

Descritores: Recém-Nascido; Nascimento Prematuro; Fisioterapia.

\section{Abstract}

Introduction: The newborn (NR) is classified as premature when presenting gestational age of 37 weeks and birth weight equal or below of $2.550 \mathrm{~g}$. Due to the immaturity of the respiratory system, the neonate is subject to present several complications, among them, the respiratory, which causes your extension in neonatal intensive care unit (UNIT). The physiotherapy respiratory is of great importance in the treatment and recovery of $\mathrm{RN}$ by applying techniques of bronchial hygiene $(\mathrm{BH})$. The study had as objectives investigate the effects of respiratory physiotherapy in newborn premature published in the scientific literature. Materials and methods: This is a revision integrative fulfilled in the database Virtual Health Library LILACS, Medline, SciELO, SCOPUS e ISI Web of Knowledge including articles published in the period of 2007-2015. Eight articles were included in this review. Results and Discussion: The performance of respiratory physiotherapy was analyzed by the effects of the application of techniques HB most used in newborn preterm (NRPT), can highlight tapotement, vibro, postural drainage and aspiration. Were realized comparisons to test the effectiveness and the possible side effects that could alter the functioning of the respiratory mechanics NR. The studies showed effectiveness of physiotherapy respiratory and effects of maneuvers respiratory condition risk of neonate. Conclusion: The Physiotherapy has a role important in the care of newborn preterm but needs further study that proving its efficiency and its importance in improving the condition of life of the newborn.

Descriptors: Infant, Newborn; Premature Birth; Physical Therapy Specialty.

\section{Resumen}

Introducción: El recién nacido (RN) es clasificado como prematuro cuando presenta edad gestacional inferior a 37 semanas y peso de nacimiento igual o inferior a $2.550 \mathrm{~g}$. Debido a la inmadurez del sistema respiratorio, el neonato está sujeto a presentar diversas complicaciones, entre ellas, las respiratorias, lo que ocasiona su prolongación en la unidad de terapia intensiva neonatal (UTIN). La fisioterapia respiratoria es de gran importancia en el tratamiento y recuperación del RN a través de la aplicación de técnicas de higiene bronquial (HB). El estudio tuvo como objetivo investigar los efectos de la fisioterapia respiratoria en el recién nacido prematuro publicados en la literatura científica. Métodos: es tratado de una la revisión que integrativa logró en las bases de biblioteca de Virtual de datos en la salud, Lilacs, Medline, SciELO, SCOPUS y Web of Science de conocimientos, publicos en el período de 2007 el 2015. Los artículos incluidos en esta revista. Resultados y hechos: el rendimiento de la fisioterapia de respiración fue analizado por los efectos de la puesta en práctica de las técnicas de HB que mayor cantidad usó en el precurso nacido - término (RNPT), podía separar el tapotaje, el vibrocompresión, postural de drenaje y aspiración. Las comparaciones seguras consumadas para demostrar que la eficacia y los efectos secundarios modifiquen la cuenta de la mecánica de respiración de RN. Los estudios mostraron la efectividad de la fisioterapia respiratoria y los efectos de las maniobras en la condición respiratoria del neonato de riesgo. Conclusión: La fisioterapia tiene un papel importante en el cuidado al recién nacido pre-término, pero necesita más estudios que demuestren su eficacia y su importancia en la mejora de la condición de vida del neonato.

Descriptores: Recién Nacido; Nacimiento Prematuro; Fisioterapia.

\section{INTRODUÇÃO}

A Organização Mundial de saúde classifica como prematuro o recém-nascido (RN) com idade gestacional inferior a 37 semanas e com peso de nascimento igual ou abaixo de 2.500 gramas, e são subdivididos em RN de muito baixo peso (inferior a 1500 gramas) e RN de extremo baixo peso (peso de nascimento inferior a 1000 gramas) $)^{1,2}$.

No Brasil, em 2012, 340.000 bebês nasceram prematuros, o que significa que nascem 931 prematuros por dia ou 40 por hora, indicando $12,4 \%$ a taxa de prematuridade, sendo o dobro quando comparado ao índice de alguns países europeus ${ }^{3}$.

Vários fatores são determinantes para prematuridade, como: doenças crônicas préexistentes, síndromes hipertensivas e hemorrágicas da gestação, alterações placentárias, excesso de 
líquido amniótico, idade materna, infecções maternas, primiparidade, realização do pré-natal, ganho de peso ${ }^{4,5}$.

As afecções respiratórias são uma das principais causas da mortalidade no período neonatal com um grande número de registros no país ${ }^{6}$. Essas complicações ocorrem na fase aguda da doença, acometendo principalmente aos recém-nascidos prétermos $\left(\right.$ RNPT) ${ }^{7,8}$.

As doenças respiratórias neonatais se manifestam no prematuro nas primeiras horas de vida devido à imaturidade pulmonar, com isso, os bebês precisam do auxílio do suporte ventilatório por longos períodos, o que ocasiona um maior tempo de internação na unidade de terapia intensiva neonatal (UTIN $)^{9}$. Devido a essa imaturidade, há comprometimento da eliminação de secreções das vias áreas, como pouca eficiência da mecânica respiratória, vias áreas mais estreitas e imaturidade do mecanismo de tosse ${ }^{10,11}$.

A atuação da fisioterapia respiratória na unidade de terapia intensiva neonatal é recente e acontece através da necessidade de tratar as doenças respiratórias e prevenir as complicações advindas das patologias. Ela tem como objetivo a eliminação das secreções, melhorar a ventilação pulmonar, aumentar o trabalho respiratório, promover a reexpansão pulmonar, melhorar a oxigenação e trocas gasosas, aumentar a mobilidade torácica, aumentar a força muscular respiratória, evitar a obstrução brônquica, hiperinsuflação pulmonar, promover a independência respiratória funcional e acelerar a recuperação do neonato ${ }^{12-16}$.

O acompanhamento fisioterapêutico tem um papel primordial no cuidado ao RNPT, possibilitando através da manobra de higiene brônquica (HB), a estabilidade nas variáveis hemodinâmicas, como na freqüência cardíaca, manutenção funcional da circulação cerebral, mantém as vias áreas com fluxo menos turbulento e com o mínimo de secreção, permitindo um aumento na permeabilidade $\mathrm{e}$ diminuição de fatores intrínsecos das vias áreas que favorecem para o aumento da resistência pulmonar ${ }^{17,18}$.

Diversas técnicas da fisioterapia podem ser utilizadas no tratamento do RNPT, em específico as manobras de higiene brônquica, como a drenagem postural, tapotagem, vibração, compressão, bag squeezing, aumento do fluxo expiratório (AFE) e aspiração ${ }^{19}$.

Diante de todos os benefícios da fisioterapia respiratória, há controvérsias sobre a sua eficácia no RNPT. Com isso, se faz necessário entender a atuação fisioterapêutica no neonato e analisar a forma de aplicação das manobras de higiene brônquica e identificar qual delas melhor se aplica ao estado clínico do neonato prematuro.

Portanto, é fundamental que se realizem estudos no sentido de avaliar a atenção da fisioterapia respiratória em bebês de risco com intuito de aprimorá-los, para que, de fato, essa prática alcance seus objetivos.

Nesse contexto, a partir de uma revisão integrativa, objetivou-se caracterizar as publicações atuais sobre a atuação da fisioterapia respiratória em recém-nascidos pré-termos e analisar seus benefícios e efeitos adversos à saúde do neonato sob cuidados especiais.

\section{MATERIAL E MÉTODO}

Trata-se de estudo de revisão exploratório, descritivo e retrospectivo, desenvolvido de forma sistematizada. Este se caracteriza como uma análise ampla da literatura sobre um assunto específico com o intuito de construir uma conclusão síntese de estudos realizados separadamente, mas que investigam problemas idênticos ou similares ${ }^{20,21}$.

Para a realização deste estudo foram seguidas às seis fases do processo de elaboração da revisão: elaboração da pergunta norteadora, busca/amostragem na literatura, coleta de dados, análise crítica dos estudos incluídos, discussão dos resultados e apresentação da revisão ${ }^{22}$.

No sentido de direcionar a revisão formularam-se as seguintes perguntas norteadoras: Quais técnicas da fisioterapia respiratória são mais utilizadas no RNPT? Quais seus benefícios e efeitos adversos para o desenvolvimento do RNPT?

A busca pelos artigos ocorreu no período de abril a outubro de 2015 e foi realizada nas bases de dados eletrônicas nacionais e internacionais na área de ciências da saúde: Biblioteca Virtual em Saúde (BVS), Literatura Latino-Americana e do Caribe (LILACS), Literatura Internacional em Ciências da Saúde (Medline), Scientific Electronic Library Online (SciELO), SCOPUS e Isi Web of Knowledge.

A seleção dos artigos seguiu os seguintes critérios de inclusão: artigos publicados em português, inglês e espanhol, textos disponíveis nas bases de dados selecionadas e abordagem do tema da pesquisa no contexto hospitalar. Como critérios de exclusão: artigos não disponíveis na íntegra no momento da pesquisa e que não apresentassem abordagem do tema no contexto hospitalar. Para os critérios de achados foram utilizados os Descritores em Ciências da Saúde (DeCS/MeSH): "Fisioterapia respiratória (Respiratory Physiotherapy)", "Atuação fisioterapêutica (Physiotherapeutic)", "Recémnascido prematuro (Premature newborn)" e "Unidade de terapia intensiva neonatal (Neonatal intensive care unit)", pesquisados juntos e encontrados no título dos artigos em todas as bases de dados.

\section{RESULTADOS E DISCUSSÃO}

Os artigos selecionados conforme autor, ano, título e delineamento da pesquisa estão apresentados na Tabela 1. 
Tabela 1. Artigos selecionados conforme autor, ano, título e delineamento da pesquisa

\begin{tabular}{|c|c|c|c|}
\hline Autor/Ano & Título do artigo & $\begin{array}{l}\text { Tipo de } \\
\text { estudo }\end{array}$ & Amostra \\
\hline $\begin{array}{c}\text { Nicolau e Lahóz }{ }^{19} \\
2007\end{array}$ & $\begin{array}{c}\text { Fisioterapia respiratória em } \\
\text { terapia intensiva pediátrica e } \\
\text { neonatal: uma revisão } \\
\text { baseada em evidências }\end{array}$ & $\begin{array}{l}\text { Revisão } \\
\text { sistemática }\end{array}$ & $\begin{array}{l}\text { Recém- } \\
\text { nascidos e } \\
\text { crianças }\end{array}$ \\
\hline $\begin{array}{c}\text { Nicolau e Falcão }{ }^{23} \\
2010\end{array}$ & $\begin{array}{c}\text { Influência da fisioterapia } \\
\text { respiratória sobre a função } \\
\text { cardiopulmonar em recém- } \\
\text { nascidos de muito baixo peso }\end{array}$ & $\begin{array}{l}\text { Estudo } \\
\text { clínico } \\
\text { prospectivo }\end{array}$ & $\begin{array}{l}42 \text { Recém- } \\
\text { nascidos } \\
\text { pré-termo }\end{array}$ \\
\hline $\begin{array}{l}\text { Souza e Moran }{ }^{24} \\
2013\end{array}$ & $\begin{array}{l}\text { Fisioterapia respiratória em } \\
\text { recém-nascido pré-termo: } \\
\text { ensaio clínico randomizado. }\end{array}$ & $\begin{array}{c}\text { Ensaio } \\
\text { clínico } \\
\text { randomizado } \\
\end{array}$ & $\begin{array}{l}43 \text { Recém- } \\
\text { nascidos } \\
\text { pré-termo }\end{array}$ \\
\hline $\begin{array}{l}\text { Antunes et al. }{ }^{25} \\
\quad 2006\end{array}$ & $\begin{array}{l}\text { Efeitos da fisioterapia } \\
\text { respiratória convencional } \\
\text { versus aumento do fluxo } \\
\text { expiratório na saturação de } \\
\text { O2, freqüência cardíaca e } \\
\text { freqüência respiratória, em } \\
\text { prematuros no período pós- } \\
\text { extubação } \\
\end{array}$ & $\begin{array}{c}\text { Estudo } \\
\text { prospectivo } \\
\mathrm{e} \\
\text { randomizado }\end{array}$ & $\begin{array}{l}\text { 40 Recém- } \\
\text { nascidos } \\
\text { prematuros }\end{array}$ \\
\hline $\begin{array}{c}\text { Nicolau e Falcão }{ }^{26} \\
2007\end{array}$ & $\begin{array}{c}\text { Efeitos da fisioterapia } \\
\text { respiratória em recém- } \\
\text { nascidos: análise crítica da } \\
\text { literatura }\end{array}$ & $\begin{array}{c}\text { Revisão } \\
\text { sistemática }\end{array}$ & $\begin{array}{l}\text { Recém- } \\
\text { nascidos }\end{array}$ \\
\hline $\begin{array}{c}\text { Santos et al. }{ }^{27} \\
2009\end{array}$ & $\begin{array}{c}\text { Efeitos de técnicas de } \\
\text { desobstrução brônquica na } \\
\text { mecânica respiratória de } \\
\text { neonatos prematuros em } \\
\text { ventilação pulmonar } \\
\text { mecânica } \\
\end{array}$ & $\begin{array}{l}\text { Ensaio } \\
\text { clínico não } \\
\text { randomizado } \\
\text { crossover }\end{array}$ & $\begin{array}{l}18 \text { Recém- } \\
\text { nascidos } \\
\text { pré-termo }\end{array}$ \\
\hline $\begin{array}{c}\text { Martins e Segre }^{28} \\
2010 \\
\end{array}$ & $\begin{array}{c}\text { Fisioterapia respiratória em } \\
\text { neonatologia: importância e } \\
\text { cuidados }\end{array}$ & $\begin{array}{c}\text { Revisão } \\
\text { sistemática }\end{array}$ & Neonatos \\
\hline $\begin{array}{c}\text { Johnston et al. }{ }^{29} \\
2012\end{array}$ & $\begin{array}{c}\text { Recomendação brasileira de } \\
\text { fisioterapia respiratória em } \\
\text { unidade de terapia intensiva } \\
\text { pediátrica e neonatal }\end{array}$ & $\begin{array}{l}\text { Revisão } \\
\text { sistemática }\end{array}$ & Neonatos \\
\hline
\end{tabular}

Este estudo avaliou através da seleção de artigos, a eficácia e os efeitos adversos das técnicas de higiene brônquica (HB) mais utilizadas em recémnascidos pré-termos (RNPT) internados na unidade de terapia intensiva neonatal.

Das diversas manobras de $\mathrm{HB}$, esse estudo teve como objetivo analisar as técnicas mais convencionais, dentre elas, destaca-se: aspiração, tapotagem (ou percussão), vibrocompressão, drenagem postural (ou posicionamento) e aumento do fluxo expiratório (AFE).

As técnicas de desobstrução brônquica têm como objetivo mobilizar e eliminar as secreções das vias áreas, melhorar relação ventilação/perfusão, reduzir o trabalho respiratório, diminuir áreas de shunt e reduzir obstrução brônquica. Segundo Piva ${ }^{22}$, as manobras de higiene brônquica são necessárias para melhorar a função pulmonar. Além dos efeitos benéficos para a melhora da mecânica respiratória do RNPT, é preciso analisar cuidadosamente os possíveis efeitos adversos que possam surgir durante a realização das técnicas da fisioterapia respiratória.

Alguns autores não indicam as manobras de higiene brônquica em RNPT com peso de nascimento inferior a 1500 gramas nos primeiros três dias de vida, por conta da probabilidade de apresentarem hemorragia intracraniana ${ }^{23,24}$. As manobras que podem interferir no aumento da Pressão intracraniana (PIC) de forma direta e indireta são: a compressão e descompressão do tórax, drenagem postural, percussão torácica e aspiração endotraqueal, sendo a última, a forma que mais eleva a PIC, causando aumento na pressão intratorácica através da tosse e com isso diminuindo o retorno venoso central ${ }^{30}$.

A aspiração endotraqueal é um procedimento invasivo bastante utilizado em recém-nascidos sob uso de via aérea artificial e ventilação mecânica internados na unidade de terapia intensiva neonatal. Ele é indicado para remoção de secreções, na presença de ruídos adventícios, sons audíveis durante a respiração com uma tosse ineficaz ${ }^{31}$. É adequado para melhorar as trocas gasosas; melhora da complacência pulmonar e diminuição da resistência das vias aéreas ${ }^{32}$.

Diversos estudos mostram que a aspiração pode gerar efeitos adversos ao RNPT, dentre eles, alterações cardiovasculares, gerado pela hipoxemia e por alterações no sistema nervoso autônomo, através da estimulação de receptores simpáticos e parassimpáticos, que respectivamente, levam secundariamente ao aumento da pressão arterial e a bradiarritmia. Outras alterações são possíveis observar, como o aumento do fluxo sanguíneo cerebral, como a queda acentuada da saturação periférica de oxigênio $\left(\mathrm{SpO}_{2}\right)^{33-35}$.

Levando em conta os efeitos adversos advindos da aspiração, o mesmo deve ser realizado apenas quando houver indicação ou sinais de secreção e não de forma rotineira. A duração do procedimento não deve exceder 15 segundos e a frequiência de inserções da sonda não pode ultrapassar 3 repetições, evitando assim possíveis agravos ao RNPT ${ }^{36}$.

A tapotagem tem como indicações pacientes hipersecretivos, atelectasiados e com doenças musculares pulmonares crônicas ${ }^{37}$. Sua eficácia é questionada por diversos autores que levam em consideração os seus efeitos adversos que superam os seus efeitos benéficos, dentre eles, pode-se destacar: hipoxemia intensa ${ }^{38,39}$, fratura de $\operatorname{costelas}^{40}$, lesões cerebrais $^{38,41}$. O RN possui um tórax flexível, com dimensões diminuídas, com isso, o efeito mecânico da tapotagem é menor, o que necessitaria que uma força maior fosse aplicada para que ocorresse o desprendimento das secreções ${ }^{38,40,42}$.

$\mathrm{O}$ estudo de Gava e Picanço ${ }^{42}$ aponta que a tapotagem por si só não é capaz de promover eliminação das secreções, mas aumenta a velocidade de eliminação do muco ${ }^{37,43,44}$. Retificando o estudo anterior, Crane et al. ${ }^{44}$ observaram em sua pesquisa realizada com 24 RNPT com síndrome do desconforto respiratório e em oxigenoterapia, sendo que 16 neonatos estavam sob uso da ventilação mecânica. Os autores compararam a tapotagem e a vibração em duas posições de drenagem postural. Verificaram que houve uma tendência ao aumento dos valores da pressão arterial de oxigênio $\left(\mathrm{PaO}_{2}\right)$ somente no grupo que recebeu a tapotagem. Observaram também que em todos os grupos houve um aumento da frequiência cardíaca e respiratória e da pressão arterial sistólica. No estudo de Duara et 
al. ${ }^{45}$, com 6 RNPT, foi aplicada tapotagem em tempo de $0.5,1.5$ e 2.5 minutos, com intervalos de duas horas e obteve como resultado uma piora dos níveis de oxigenação arterial em todos os grupos, menos naquele que recebeu a tapotagem por 2,5 minutos, no qual houve aumento nos valores de $\mathrm{PaO}_{2}$.

Pesquisa conduzida por Tudehope e Bagley ${ }^{46}$ com 15 recém-nascidos com síndrome do desconforto respiratório sob uso da ventilação mecânica, compararam três técnicas: tapotagem com punhos em contato, tapotagem com máscara de ventilação com pressão positiva e vibração com escova de dente elétrica. As manobras foram feitas em quatro posições de drenagem postural, seguida de aspiração traqueal. Foi observado no estudo que os neonatos demonstraram melhor tolerância às manobras de tapotagem do que a de vibração, como também os níveis de oxigenação permaneceram instáveis em todos os grupos após a aspiração.

A vibração consiste do movimento oscilatório realizado com as mãos durante o tempo expiratório; tem como objetivo o deslocamento das secreções, podendo ser associada à compressão torácica, que ocorre durante a fase expiratória do ciclo respiratório, de forma lenta e constante, facilitando a mobilização de secreções das vias áreas mais calibrosas ${ }^{47}$. A associação das técnicas de vibração mecânica e compressão torácica denominam-se vibrocompressão. A técnica é utilizada em neonatos com dificuldade de mobilização de secreção e que não suportam a tapotagem. Está contra-indicada em recém-nascido de extremo baixo peso, hemorragia pulmonar e presença de enfisema intersticial não drenado ${ }^{48}$.

Para Cuello et al. ${ }^{49}$ a técnica é mais eficaz quando aplicada na região anterior da parede torácica. Devido à pressão exercida no tórax, recomenda-se que durante sua aplicação seja verificado o movimento do tórax com o intuito de não alterar os movimentos do gradil costal, podendo levar a alteração da normalidade dos movimentos respiratórios.

Curran e Kachoyeanos ${ }^{50}$, em grupo com seis neonatos, compararam a vibração com escova de dente elétrica e tapotagem com bico de mamadeira acolchoado e verificaram uma maior eficiência da vibração quanto à melhora dos parâmetros de oxigenação arterial, ausculta pulmonar e perfusão periférica. Observou que todos os pacientes apresentaram taquicardia, taquipnéia e agitação durante e logo depois dos procedimentos.

A drenagem postural é uma técnica que se utiliza da ação da gravidade, por meio do posicionamento invertido, mobilizando as secreções para as vias áreas centrais, onde serão removidas através da tosse. Além de seu benefício em deslocar a secreção para uma região mais superior da árvore brônquica, ela promove a melhora da relação ventilação/perfusão ${ }^{51}$. Ela pode vir associada a outras técnicas, como a vibração ou percussão ou ser aplicada isoladamente.

No estudo de Finer e Boyd ${ }^{52}$ realizado com 20 recém-nascidos com insuficiência respiratória, os autores observaram que após o tratamento realizado com drenagem postural de forma isolada e drenagem associada à tapotagem, houve aumento dos níveis de $\mathrm{PaO}_{2}$ somente nos neonatos que receberam as duas manobras. No recém-nascido pré-termo, o posicionamento é fundamental nos primeiros dias de vida, pois permitirá melhores condições biomecânicas ao segmento tóraco-abdominal ${ }^{29}$. Para cada área do pulmão há uma postura de drenagem específica, que associada ao posicionamento mais à ação da gravidade, facilitará a drenagem das secreções.

Em relação às posições, vários autores através de seus estudos, verificaram que a postura prona é mais eficaz do que a supina em RN com insuficiência respiratória, pois nessa posição a caixa torácica torna-se mais estável, o que favorece os músculos respiratórios a expandir o tórax, além de proporcionar aumento do volume corrente com melhora da oxigenação arterial, na relação ventilação/perfusão e da complacência pulmonar ${ }^{29,53}$.

Goto et al. ${ }^{54}$ avaliaram a variabilidade da frequência cardíaca em posição prona e supina em RNPT e concluiram que na posição prona a frequência cardíaca se manteve mais estável. No recém-nascido em decúbito lateral há uma diminuição da ventilação pulmonar no pulmão dependente. Esse recurso tem sido utilizado para hipoventilação localizada, onde o neonato fica em decúbito lateral com o lado comprometido na posição dependente; pneumotórax unilateral não hipertensivo; em casos de enfisema intersticial localizada ${ }^{19}$. No neonato prematuro, a postura de Trendelemburg está contraindicada, pois o controle do fluxo sanguíneo cerebral é limitado e especificamente nessa posição, ocorrem vibrações no fluxo sanguíneo sistêmico e cerebral, predispondo a ocorrência tanto de lesões isquêmicas como hemorrágicas s $^{37,47,51,53}$.

No estudo de Lanza et al. ${ }^{55}$, neonatos com bronquiolite viral aguda foram divididos em 3 grupos, sendo eles: Vibrocompressão + Drenagem Postural (VC+DP), Tapotagem + Drenagem Postural (TAP+DP) e Aspiração (ASP). Os resultados mostraram que o grupo que recebeu a $\mathrm{VC}+\mathrm{DP}$ e TAP+DP apresentaram diminuição do desconforto respiratório, maior quantidade de secreção aspirada e melhora na ausculta pulmonar em relação ao grupo ASP.

O aumento do fluxo expiratório (AFE) é uma manobra desobstrutiva que mobiliza a caixa torácica através da compressão do tórax com apoio do abdome e tem como finalidade aumentar o fluxo aéreo expiratório, deslocando secreções brônquicas 
em grande velocidade (AFE rápida) ou acelerando o fluxo de forma mais prolongada, mobilizando, desta forma, a secreção com o fluxo de ar da região distal para proximal (AFE lenta) $)^{25}$.

O AFE tem contraindicações em RN com hipertensão intracraniana, osteopenia da prematuridade, hemorragia Peri e intraventricular, distúrbios hemorrágicos e instabilidade hemodinâmica ${ }^{25}$.

Antunes et al. ${ }^{25}$, ao estudar 40 recémnascidos prematuros, utilizou em um grupo $(n=20)$ a fisioterapia respiratória convencional (FRC), composta pela drenagem postural e tapotagem e no outro grupo $(\mathrm{n}=20)$, aumento do fluxo expiratório (AFE). Observou que houve aumento da $\mathrm{SaO}_{2} \mathrm{em}$ ambos os grupos e que a frequência cardíaca não sofreu alteração apenas no grupo que recebeu a AFE, o que considera que a técnica seja menos estressante ao $\mathrm{RN}$.

Outras técnicas fisioterapêuticas desenvolvidas recentemente buscam remover as secreções sem a realização de procedimentos invasivos, como a glossopulsão retrógrada (GPR), a desobstrução rinofaríngea retrógrada + instilação $(\mathrm{DRR}+\mathrm{I})$ e a drenagem autógena assistida (DAA), terapia expiratória manual passiva (TEMP) e reequilíbrio tóraco-abdominal (RTA $)^{56}$.

O acompanhamento fisioterapêutico contribui para melhora das trocas gasosas, relação ventilação/perfusão e complacência pulmonar; diminuição da resistência das vias áreas; aumento da velocidade de eliminação do muco, mantendo as vias áreas com um fluxo menos turbulento e melhora das condições biomecânicas ao segmento tóracoabdominal $^{48}$. Em relação aos riscos de efeitos adversos encontrados nos estudos destacam-se as alterações cardiovasculares; aumento do fluxo sanguíneo cerebral; fratura de costelas e lesões cerebrais.

Diante dos estudos analisados, não se pode descartar ou ignorar a importância da fisioterapia respiratória na melhora do funcionamento da mecânica respiratória do recém-nascido prematuro. Com isso, se faz necessário que as técnicas sejam revistas e outras, não tão convencionais, sejam utilizadas e associadas juntamente com as manobras de expansão pulmonar e exercícios respiratórios. A escolha de técnicas fisioterapêuticas inadequadas pode contribuir para que o RNPT esteja mais vulnerável as alterações clínicas, aumentando assim o risco a condições adversas.

\section{CONCLUSÃO}

Diante dos estudos sobre as técnicas de fisioterapia respiratória, é possível analisar seus benefícios na funcionalidade da mecânica respiratória do recém-nascido prematuro, através da manobras de higiene brônquica que auxiliam na eliminação de secreção das vias aéreas.

As técnicas mais convencionais destacadas no estudo foram à aspiração, tapotagem, vibrocompressao, drenagem postural e AFE, podendo ser realizadas isoladamente ou associadas com outras técnicas. As manobras foram efetivas ao recémnascido, confirmando que a fisioterapia respiratória é essencial e necessária para melhora do quadro respiratório do neonato na unidade de terapia intensiva. Contudo, as técnicas devem ser aplicadas de forma cautelosa e analisando o estado atual do neonato. Com isso, se faz necessária avaliação minuciosa do fisioterapeuta, observando quais manobras melhores se aplicam para condição do recém-nascido.

A presente revisão da literatura corroborou que a atuação da fisioterapia respiratória necessita de mais estudos que provem seus efeitos no RNPT, sendo importante para manutenção da ventilação adequada e prevenção de complicações relacionadas à imaturidade do sistema respiratório e aos riscos do tempo de internação do neonato na UTIN. Ademais, a fisioterapia tem ampla forma de atuação e necessita que outras técnicas não convencionais sejam utilizadas para melhor assistir ao recém-nascido prematuro, contribuindo para o seu desenvolvimento respiratório e neuropsicomotor.

\section{REFERÊNCIAS}

1. Nikolovi V. Congenital malformations and perinatal mortality at the Saint Antoine University Obstetric. Gynecologic Clinic. 1989;28(1):36-4.

2. Oliveira RMS, Franceschini SCC, Priore SE. Avaliação antropométrica do recém-nascido prematuro e/ou pequeno para a idade gestacional. Rev Bras Nutr Clín. 2008;23(4):298-30.

3. Calafiori L. Taxa de prematuridade no Brasil. 2014. Disponível em: <http://www.uicamp.br/ unicamp/noticias/2014/11/14/brasil-tem-40-partos -prematuros-por-hora>. Acesso em: $17 \mathrm{de}$ mai.2016.

4. Benício MHD, Monteiro CA, Souza JMP, Castilho EA, Lamonica IMR. Análise de fatores de risco para o baixo peso ao nascer em nascidos vivos do município de São Paulo. Rev Saúde Pública. 1985;19(4):311-20.

5. Ramos HAC, Cuman RKN. Fatores de risco para prematuridade: pesquisa documental. Rev Enferm. 2009;13(2):297-304.

6. Carvalho ML, Silver LD. Confiabilidade da declaração da causa básica de óbitos neonatais: implicações para o estudo da mortalidade prevenível. Rev Saúde Pública 1995;29(5):342-48.

7. Mendonça EF, Goulart EMA, Machado JAD. Confiabilidade da declaração de causa básica de mortes infantis em região metropolitana do 
sudeste do Brasil. Rev Saúde Pública 1994;28(5):385-91.

8. Ferrari LSL, Brito ASJ, Carvalho ABR, Gonzáles MRC. Mortalidade neonatal no município de Londrina, Paraná, Brasil, nos anos 1994,1999 e 2002. Cad Saúde Pública 2006; 22(5):1063-71.

9. Ministério da Saúde. Atenção à saúde do recémnascido. Guia para os profissionais de saúde. 2. ed. Brasília-DF; 2012. p.11-38.

10.Lewis JA, Lacey JL, Henderson-Smart DJ. A review of chest physiotherapy in neonatal intensive care units in Australia. J Paediatr Child Health. 1992;28(4):297-300.

11.Graziela MM, Abreu CF, Miyoshi MH. Papel da fisioterapia respiratória nas doenças respiratórias neonatais. Clin Perinatol. 2010;1(1):145.

12.Etches PC, Scott B. Chest physiotherapy in the newborn: effect on secretions removed. Pediatrics. 1978;62(5):713-15.

13. All-Alaiyan S, Dyer D, Khan B. Chest physiotherapy and pós-extubation atelectasis in infants. Pediatric Pulmonol. 1996;21(4):227-30.

14.Azeredo CAC. Fisioterapia respiratória atual. Rio de Janeiro: Edusuam; 1986.

15. Azeredo CAC. Fisioterapia respiratória moderna. São Paulo: Editora Manole; 1993.

16. Costa D. Fisioterapia respiratória básica. São Paulo: Editora Atheneu; 1999.

17.Flenady VJ, Gray BH. Chest physiotherapy for preventing morbidity in babies being extubated from mechanical ventilation. Cochrane Database Syst Rev 2000;(2):CD000283.

18. Guy P. Groupe d' Étude Pluridisciplinaire Stéthacoustique. Novas técnicas de fisioterapia. 2013. Available from:<http://www.postiaux.com/ $\mathrm{pt} /$ methode.html>. Acesso em 17 de mai.2016.

19. Nicolau CM, Lahóz AL. Fisioterapia respiratória em terapia intensiva pediátrica e neonatal: uma revisão baseada em evidências. Pediatria. 2007;29(3):216-21.

20.Barbosa LR, Melo MRAC. Relações entre qualidade da assistência de enfermagem: revisão integrativa da literatura. Rev Bras Enferm. 2012;61(3):366-70.

21.Souza MT, Silva MD, Carvalho R. Revisão integrativa: o que é e como fazer. Einstein. 2010;8(1):102-6.

22.Piva JP, Garcia PCR. Insuficiência Respiratória Aguda. IN: Piva JP, Carvalho P, Garcia PCR. Terapia Intensiva em Pediatria. Medsi: Rio de Janeiro; 2009.p.163.

23. Nicolau CM, Falcão MC. Influência da fisioterapia resiratória sobre a função cardiopulmonar em recém-nascido de muito baixo peso. Rev Paul Pediatr. 2010; 28(2):170-75.

24. Souza JAQ, Moran CA. Fisioterapia respiratória em recém-nascidos pré-termo: ensaio clínico randomizado. Rev Bras Med. 2013;49(11):434-38.
25. Antunes LCO, Silva EG, Bocardo P, Daher DR, Faggiotto RD, Rugolo LMSS. Efeitos da fisioterapia convencional versus aumento do fluxo expiratório na saturação de $\mathrm{O} 2$, frequiência cardíaca e frequiência respiratória em prematuro no período pós-extubação. Rev bras fisioter. 2006; 10(1):97-103.

26.Nicolau CM, Falcão MC. Efeitos da fisioterapia respiratória em recém-nascidos: análise crítica da literatura. Rev Paul Pediatria. 2007;25(1):72-5.

27. Santos MLM, Souza LA, Batiston AP, Palhares DB. Efeitos de técnicas de desobstrução brônquica na mecânica respiratória de neonatos prematuros em ventilação pulmonar mecânica. Rev. bras. ter. intensiva . 2009;21(2):183-89.

28. Martins AP, Segre CAM. Fisioterapia respiratória em neonatologia: importância e cuidados. Pediatr mod. 2010;46(2):56-60.

29.Johnston C, Zanetti NM, Comaru T, Ribeiro SNS, Andrade LB, Santos SLL. I Recomendação brasileira de fisioterapia respiratória em unidade de terapia intensiva pediátrica e neonatal. Rev bras ter intensiva. 2012;24(2):119-29.

30.De Paula LC, Ceccon ME. Análise comparativa randomizada entre dois tipos de sistema de aspiração traqueal em recém-nascidos. Rev Assoc Med Bras. 2010;56(4):434-39.

31.Gonçalves RL, Tsuzuki, LM, Carvalho, MGS. Aspiração endotraqueal em recém-nascidos intubados: uma revisão integrativa da literatura. Rev bras ter intensiva. 2015;27(3):284-92 .

32.American Association for Respiratory Care. AARC Clinical Practice Guidelines. Endotracheal suctioning of mechanically ventilated patients with artificial airways. Respir care. 2010;55(6):758-64.

33. Rosa FK, Roese CA, Savi A, Dias AS, Monteiro MB. Comportamento da mecânica pulmonar após a aplicação de protocolo de fisioterapia respiratória e aspiração traqueal em pacientes com ventilação mecânica invasiva. Rev bras ter intensiva. 2007;19(2):170-75.

34.Pederson CM, Rosendahl-Nielsen M, Hjermind J, Egerod I. Endotracheal suctioning of the adult intubated patiente-what is the evidence? Intensive Crit Care Nurs. 2009;25(1):21-30

35. Gonçalves RL, Tsuzuki LM, Carvalho MGS. Aspiração endotraqueal em recém-nascidos intubados: uma revisão integrativa da literatura. Rev bras ter intensiva. 2015;27(3):284-92.

36. Selsby DS. Chest physiotherapy. BMJ. 1989; 298(6673):541-42.

37.Ferreira ACL, Troster EJ. Atualização em terapia intensiva pediátrica. Rio de Janeiro: Interlivros; 1996.

38. Holloway R, Adams EB, Desai SD, Thambiran AK. Effect of chest physiotherapy on blood gases of neonates treated by intermittent positive pressure respiration. Thorax. 1996;24(4):421-26. 
39. Wood BP. Infant ribs: gereralized periosteal reaction resulting from vibrator chest physiotherapy. Radiology. 1987;162(3):811-12.

40. Raval D, Yeh TF, Mora A. Chest physiotherapy in preterm infants wih RDS in the first 24 hours of life. J Perinatol. 1987;7(4):301-4

41.Juliani RCTP, Lahóz ALC, Nicolau CM, Paula LCS, Cunha MT. Fisioterapia nas unidades de terapia intensiva pediátrica e neonatal. Programa Nacional de educação continuada em Pediatria. PRONAP 2003/2004; 70: 9-24.

42. Gava MV, Picanço PSA. Fisioterapia pneumológica. São Paulo: Manole; 2007.

43.Figueira F. Pediatria. 3. ed. Rio de Janeiro: Guanabara Koongan; 2004.

44. Crane LD, Zombek M, Krauss NA, Auld PA. Comparison of chest physiotherapy techniques in infants with RDS. Pediatr Res.1978;12:559A.

45.Duara S, Bessard K, Keszler L. Evaluation of different percussion time intervals at chest physiotherapy on neonatal pulmonary function parameters. Pediatr Res. 1983; 17:310A.

46. Tudehope DI, Bagley C. Techniques of physiotherapy in intubated babies with the respiratory distress syndrome. Aust Paediatr J. 1980;16(4):226-28.

47. Selestrin CC, Oliveira AG, Ferreira C, Siqueira AAF, Abreu LC, Murad N. Avaliação dos parâmetros fisiológicos em recém-nascidos prétermo em ventilação mecânica após procedimentos de fisioterapia neonatal. Rev bras crescimento desenvolv hum. 2007;17(1):146-55.

48. Nicolau CM. Estudo das repercussões da fisioterapia respiratória sobre a função cardiopulmonar em recém-nascido pré-termo de muito baixo peso [dissertação]. São Paulo: Faculdade de Medicina USP; 2006.

49. Cuelo AF, Arcodaci CS, Feltrim MIZ. Broncoobstrução. São Paulo: Panamericana. 1987.

50.Curran LC, Kachoyeanos MK. The effects of neonates of two methods of chest physical therapy. Mothercraft Nursing. 1979;4:309-13.

51. Oliveira LRC. Padronização do desmame da ventilação mecânica em Unidade de Terapia Intensiva: resultados após um ano. Rev bras ter intensiva. 2006;18(2):131-36.

52. Finer NN, Boyd J. Chest physiotherapy in the neonate: a controlled study. Pediatrics. 1978; 61(2):282-85.

53. Alcântara PC, Filho JOS, Lima TCP. Atuação da fisioterapia respiratória em recém-nascido com a síndrome do desconforto respiratório. Revisão da literatura. EFDeportes.2015;19(202).

54.Goto K, Maeda T, Mirmiram M; Ariagno R. Effects of prone and supine position on sleep characteristics in preterm infants. Psychiatry Clin Neurosci. 1999, 53(2):315-17.
55.Lanza FC, Gazzotti MR, Luque A, Cadrobbi C, Faria R, Solé D. Fisioterapia respiratória em lactantes com bronquiolite: realizar ou não? Mundo Saúde. 2008;32(2):183-88.

56. Haddad ER, Costa LCD, Negrini F, Sampaio LMM. Abordagens fisioterapêuticas para remoção de secreções das vias aéreas em recém-nascidos: relato de casos. Pediatria. 2006;28(2):135-40.

\section{CONFLITO DE INTERESSES}

Os autores declaram não haver conflitos de interesse.

\section{AUTOR PARA CORRESPONDENCIA}

\section{Yago Tavares Pinheiro}

yagostavares5@gmail.com

Submetido em 01/08/2018

Aceito em 04/10/2018 